Archived version from NCDOCKS Institutional Repository http://libres.uncg.edu/ir/asu/

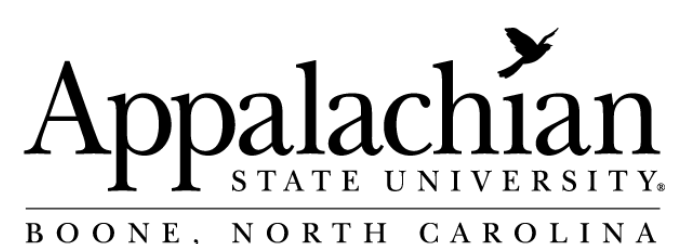

Local Presentability Of Certain Comma Categories

\author{
By: Andrew Polonsky and Patricia Johann
}

\begin{abstract}
It follows from standard results that if $A$ and $C$ are locally $\lambda$-presentable categories and $F: A \rightarrow C$ is a $\lambda$ accessible functor, then the comma category IdC $\downarrow F$ is locally $\lambda$-presentable. We show that, under the same hypotheses, $F \downarrow$ IdC is also locally $\lambda$-presentable.
\end{abstract}

Polonsky, A. \& Johann, P. (2020). Local presentability of certain comma categories. Applied Categorical Structures 28(1) (2020), pp. 135-142. Publisher version of record available at: https://link.springer.com/ article/10.1007/s10485-019-09574-w 


\title{
Local presentability of certain comma categories
}

\author{
Andrew Polonsky · Patricia Johann
}

July 15,2019

Abstract It follows from standard results that if $\mathcal{A}$ and $\mathcal{C}$ are locally $\lambda$-presentable categories and $F: \mathcal{A} \rightarrow \mathcal{C}$ is a $\lambda$-accessible functor, then the comma category $\operatorname{ld}_{\mathcal{C}} \downarrow F$ is locally $\lambda$-presentable. We show that, under the same hypotheses, $F \downarrow l_{\mathcal{C}}$ is also locally $\lambda$-presentable.

\section{Introduction}

Locally presentable categories form a robust class of categories that possess very nice properties, yet are general enough to encompass a large class of examples, including categories of models of algebraic theories and limit-sketches.

The category $F \downarrow I_{\mathcal{C}}$ plays a central role in Kelly's transfinite construction of algebras [2]. The main result of this paper gives more control over the construction when $F$ is an accessible endofunctor on a locally presentable category. Specifically, it gives the best possible bound on the number of steps required for Kelly's "algebra reflection sequence" to converge.

We assume familiarity with the notions, parameterized by a regular cardinal $\lambda$, of $\lambda$-presentable objects, locally $\lambda$-presentable and $\lambda$-accessible categories, and $\lambda$ accessible functors. We refer to the standard reference [1] for definitions and basic properties of these, which include the following:

Proposition 1 If $\mathcal{C}$ is a locally $\lambda$-presentable category, then for each object $X$ of $\mathcal{C}$, the slice categories $\mathcal{C} / X$ and $X / \mathcal{C}$ are locally $\lambda$-presentable. [1, Proposition 1.57]

Proposition 2 If $\mathcal{A}, \mathcal{B}$, and $\mathcal{C}$ are locally $\lambda$-presentable categories, and $F_{1}: \mathcal{A} \rightarrow$ $\mathcal{C}$ and $F_{2}: \mathcal{B} \rightarrow \mathcal{C}$ are $\lambda$-accessible functors that preserve limits, then the comma category $F_{1} \downarrow F_{2}$ is locally $\lambda$-presentable. [1, Exercise 2.h]

A. Polonsky, P. Johann

Department of Computer Science

Appalachian State University

E-mail: andrew.polonsky@gmail.com, johannp@appstate.edu 
Proposition 3 If $F_{1}: \mathcal{A} \rightarrow \mathcal{C}$ and $F_{2}: \mathcal{B} \rightarrow \mathcal{C}$ are $\lambda$-accessible functors, then there exists a regular cardinal $\lambda^{\prime} \geq \lambda$ such that $F_{1} \downarrow F_{2}$ is $\lambda^{\prime}$-accessible. Furthermore, if $F_{1}$ preserves $\lambda$-presentable objects, then $F_{1} \downarrow F_{2}$ is $\lambda$-accessible. [1, Proposition 2.43]

In particular, Proposition 3 entails that if $\mathcal{A}$ and $\mathcal{C}$ are locally $\lambda$-presentable categories and $F: \mathcal{A} \rightarrow \mathcal{C}$ is $\lambda$-accessible, then $\operatorname{Id}_{\mathcal{C}} \downarrow F$ is also locally $\lambda$-presentable. (For details, see Section 2 below.)

In this paper, we complement this observation with the following result:

Proposition 4 If $\mathcal{A}$ and $\mathcal{C}$ are locally $\lambda$-presentable categories and $F: \mathcal{A} \rightarrow \mathcal{C}$ is a $\lambda$-accessible functor, then $F \downarrow \mathrm{ld}_{\mathcal{C}}$ is locally $\lambda$-presentable.

In contrast to Proposition 3, Proposition 4 entails no "bump" from $\lambda$ to a larger cardinal $\lambda^{\prime}$, without any additional hypotheses on $F$ beyond $\lambda$-accessibility.

Note that if $F_{1}: \mathcal{A} \rightarrow \mathcal{C}$ and $F_{2}: \mathcal{B} \rightarrow \mathcal{C}$ are $\lambda$-accessible functors between locally $\lambda$-presentable categories, then $F_{1} \downarrow F_{2}$ will not, in general, be cocomplete, and thus will not be locally presentable.

Example 1 With $\mathcal{A}=\mathcal{B}=\mathcal{C}=$ Set, let $F_{1}=K_{1}$ and $F_{2}=K_{1+1}$ be constant functors with values $\mathbf{1}$ and $\mathbf{1}+\mathbf{1}$, respectively. The category $F_{1} \downarrow F_{2}$ has no initial object and thus is not cocomplete.

For the remainder of this paper, let $\mathcal{A}$ and $\mathcal{C}$ be locally $\lambda$-presentable categories, and let $F: \mathcal{A} \rightarrow \mathcal{C}$ be a $\lambda$-accessible functor.

\section{$2 \operatorname{Id}_{\mathcal{C}} \downarrow F$ is locally $\lambda$-presentable}

We begin by recalling that if $\mathcal{A}$ and $\mathcal{C}$ are locally $\lambda$-presentable and $F: \mathcal{A} \rightarrow \mathcal{C}$ is $\lambda$-accessible, then $\operatorname{ld}_{\mathcal{C}} \downarrow F$ is locally $\lambda$-presentable.

The fact that $I_{\mathcal{C}}$ preserves $\lambda$-presentable objects implies that $\operatorname{Id}_{\mathcal{C}} \downarrow F$ is $\lambda$ accessible by the argument given in the proof of [1, Prop. 2.43] showing that $F_{1} \downarrow F_{2}$ is $\lambda$-accessible. That proof begins by choosing a new cardinal $\lambda^{\prime} \geq \lambda$ such that $F_{1}$ and $F_{2}$ (are $\lambda^{\prime}$-accessible and) preserve $\lambda^{\prime}$-presentable objects, but subsequently only uses this information about $F_{1}$. When $F_{1}=\mathrm{Id}_{\mathcal{C}}$ we can therefore take $\lambda^{\prime}=\lambda$ and proceed as is done there. Since a category is locally $\lambda$-presentable iff it is $\lambda$-accessible and cocomplete, it remains to show that $\operatorname{Id}_{\mathcal{C}} \downarrow F$ is cocomplete. This follows immediately from the fact that $\mathrm{Id}_{\mathcal{C}}$ is cocontinuous:

Proposition 5 If $\mathcal{A}, \mathcal{B}$, and $\mathcal{C}$ are cocomplete categories, $F_{1}: \mathcal{A} \rightarrow \mathcal{C}, F_{2}: \mathcal{B} \rightarrow \mathcal{C}$, and $F_{1}$ is cocontinuous, then $F_{1} \downarrow F_{2}$ is cocomplete.

Proof Let $\mathcal{I}$ be a small category, and let $\left\{\left(X_{i}, Y_{i}, f_{i}\right)\right\}_{i \in \mathcal{I}}$, where $f_{i}: F_{1} X_{i} \rightarrow F_{2} Y_{i}$ for each $i \in \mathcal{I}$, be a diagram in $F_{1} \downarrow F_{2}$. If $\iota: i \rightarrow i^{\prime}$ in $\mathcal{I}$ then write $X_{\iota}: X_{i} \rightarrow X_{i^{\prime}}$ for the connecting morphism in $\mathcal{A}$ and $Y_{\iota}: Y_{i} \rightarrow Y_{i^{\prime}}$ for the morphism in $\mathcal{B}$ that determine the connecting morphism from $\left(X_{i}, Y_{i}, f_{i}\right)$ to $\left(X_{i^{\prime}}, Y_{i^{\prime}}, f_{i^{\prime}}\right)$ provided by the diagram.

By cocompleteness, let $X=\underline{\lim }_{i \in \mathcal{I}} X_{i}$ in $\mathcal{A}$ and $Y=\underline{\lim }_{i \in \mathcal{I}} Y_{i}$ in $\mathcal{B}$, with colimit morphisms $x_{i}: X_{i} \rightarrow X$ and $y_{i}: Y_{i} \rightarrow Y$, respectively.

Note that $\left\{F_{2} y_{i} \circ f_{i}\right\}_{i \in \mathcal{I}}$ makes $F_{2} Y$ the vertex of a cocone for $\left\{F_{1} X_{i}\right\}_{i \in \mathcal{I}}$. Since $F_{1}$ is cocontinuous, so that $F_{1} X=\varliminf_{i \in \mathcal{I}} F_{1} X_{i}$, there must therefore exist a 
unique $f: F_{1} X \rightarrow F_{2} Y$ such that $F_{2} y_{i} \circ f_{i}=f \circ F_{1} x_{i}$ for all $i \in \mathcal{I}$, i.e., such that $f: F_{1} X \rightarrow F_{2} Y$ is the vertex of a cocone for $\left\{\left(X_{i}, Y_{i}, f_{i}\right)\right\}_{i \in \mathcal{I}}$.

Now, suppose $f^{\prime}: F_{1} X^{\prime} \rightarrow F_{1} Y^{\prime}$ is another cocone for $\left\{\left(X_{i}, Y_{i}, f_{i}\right)\right\}_{i \in \mathcal{I}}$ in $F_{1} \downarrow F_{2}$ with $x_{i}^{\prime}: X_{i} \rightarrow X^{\prime}$ and $y_{i}^{\prime}: Y_{i} \rightarrow Y^{\prime}$ comprising the cocone morphisms. Let $x_{0}: X \rightarrow X^{\prime}$ and $y_{0}: Y \rightarrow Y^{\prime}$ be obtained by the colimiting properties of $X$ and $Y$, respectively. Then

- for all $i \in \mathcal{I}, x_{0} \circ x_{i}=x_{i}^{\prime}$ and $y_{0} \circ y_{i}=y_{i}^{\prime}$ by choice of $x_{0}$ and $y_{0}$;

$-f^{\prime} \circ F_{1} x_{0}=F_{2} y_{0} \circ f$ since their precompositions with each $F_{1} x_{i}$ are the same;

- $\left(x_{0}, y_{0}\right):(X, Y, f) \rightarrow\left(X^{\prime}, Y^{\prime}, f^{\prime}\right)$ is the unique morphism with the above two properties, also by choice of $x_{0}$ and $y_{0}$.

Thus $(X, Y, f)=\lim _{i \in \mathcal{I}}\left(X_{i}, Y_{i}, f_{i}\right)$ in $F_{1} \downarrow F_{2}$.

In particular, if $\mathcal{C}$ is cocomplete, then $\operatorname{Id}_{\mathcal{C}} \downarrow F$ is cocomplete.

Corollary 1 If $\mathcal{A}$ and $\mathcal{C}$ are locally $\lambda$-presentable categories, and $F: \mathcal{A} \rightarrow \mathcal{C}$ is a $\lambda$-accessible functor, then $\operatorname{Id}_{\mathcal{C}} \downarrow F$ is locally $\lambda$-presentable.

\section{$3 F \downarrow l d_{\mathcal{C}}$ is locally $\lambda$-presentable}

In this section we prove Proposition 4 , our main result. First, we verify cocompleteness. Thereafter, we define a set $\mathcal{P}$ of objects in $F \downarrow l d_{\mathcal{C}}$ and prove that they are all $\lambda$-presentable. Finally, we show that every object in $F \downarrow l_{\mathcal{C}}$ is a $\lambda$-directed colimit of elements of $\mathcal{P}$.

\subsection{Cocompleteness of $F \downarrow l d_{\mathcal{C}}$}

As noted in Proposition 5, cocompleteness of $F_{1} \downarrow F_{2}$ follows when $F_{1}$ is cocontinuous. Under the hypotheses of Proposition 4 , we know only that $F_{1}=F$ is $\lambda$-cocontinuous, i.e., preserves $\lambda$-directed colimits. Nevertheless, when $F_{2}=\operatorname{ld}_{\mathcal{C}}$ we can still compute arbitrary colimits.

Let $\left(X_{i}, Y_{i}, f_{i}\right)_{i \in \mathcal{I}}$, where $f_{i}: F X_{i} \rightarrow Y_{i}$, be a small diagram in $F \downarrow \mathbf{l d}_{\mathcal{C}}$, with connecting morphisms $\left(X_{\iota}, Y_{\iota}\right):\left(X_{i}, Y_{i}, f_{i}\right) \rightarrow\left(X_{i^{\prime}}, Y_{i^{\prime}}, f_{i^{\prime}}\right)$ whenever $\iota: i \rightarrow i^{\prime}$.

Let $X=\lim _{i \in \mathcal{I}} X_{i}, Y=\underline{\lim }_{i \in \mathcal{I}} Y_{i}$ via cocones $\left\{x_{i}: X_{i} \rightarrow X\right\}_{i \in \mathcal{I}}$ and $\left\{y_{i}:\right.$ $\left.Y_{i} \rightarrow Y\right\}_{i \in \mathcal{I}}$.

Next, let $p$ be the comparison morphism from the actual colimit of $F X_{i}$ in $\mathcal{C}$ to the cocone $\left(F X,\left\{F x_{i}\right\}_{i \in \mathcal{I}}\right)$. Similarly, let $q$ be the morphism from this colimit to the cocone $\left(Y,\left\{y_{i} \circ f_{i}\right\}_{i \in \mathcal{I}}\right)$.

Now form the pushout of $p$ and $q$. A routine calculation using the colimiting properties of $X$ and $Y$ and the fact that $Z$ is a pushout verifies that the cocone $\left\{\left(x_{i}, g \circ y_{i}\right)\right.$ :

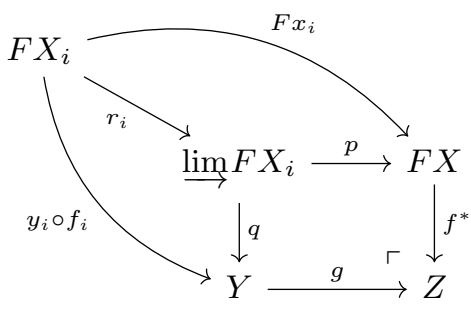
$\left.\left(X_{i}, Y_{i}, f_{i}\right) \rightarrow\left(X, Z, f^{*}\right)\right\}_{i \in \mathcal{I}}$ satisfies the universal property of the colimit. That is, $\lim _{i \in \mathcal{I}}\left(X_{i}, Y_{i}, f_{i}\right)=\left(X, Z, f^{*}\right)$. 
3.2 Presentable objects of $F \downarrow l_{\mathcal{C}}$

The $\lambda$-presentable objects of $F \downarrow l d_{\mathcal{C}}$ will be generated from a set $\mathcal{W}$ of "witnessing data". That is, each $w \in \mathcal{W}$ will determine an object $\left(A_{w}, B_{w}, f_{w}\right)$, where $f_{w}$ : $F A_{w} \rightarrow B_{w}$, in $F \downarrow l_{d_{C}}$ which we will show in this section to be $\lambda$-presentable. In Section 3.3 we will show that these objects also generate all of $F \downarrow l d_{\mathcal{C}}$ under $\lambda$-directed colimits.

Let $\mathcal{A}_{0}, \mathcal{C}_{0}$ be small sets containing a representative from each isomorphism class of $\lambda$-presentable objects in $\mathcal{A}$ and $\mathcal{C}$, respectively. Define

$$
\mathcal{W}:=\left\{(A, P, Q, p, q) \mid A \in \mathcal{A}_{0}, P \in \mathcal{C}_{0}, Q \in \mathcal{C}_{0}, p: P \rightarrow F A, q: P \rightarrow Q\right\}
$$

Since locally $\lambda$-presentable categories are locally small, $\mathcal{W}$ is a (small) set.

For each $w \in \mathcal{W}$, form the pushout

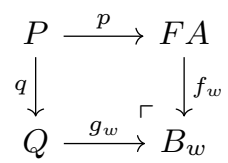

Having chosen such a triple $\left(B_{w}, f_{w}, g_{w}\right)$ for each $w \in \mathcal{W}$, and writing $A_{w}$ for the first component of $w$, define

$$
\mathcal{P}:=\left\{\left(A_{w}, B_{w}, f_{w}\right) \mid w \in \mathcal{W}\right\}
$$

Then $\mathcal{P}$ is a (small) set of objects in $F \downarrow l_{\mathcal{C}}$.

Proposition 6 Every $(A, B, f) \in \mathcal{P}$ is $\lambda$-presentable in $F \downarrow$ ld $_{\mathcal{C}}$.

Proof Let $A \in \mathcal{A}_{0}, P \in \mathcal{C}_{0}, Q \in \mathcal{C}_{0}$, and suppose $f: F A \rightarrow B$ is given by the pushout

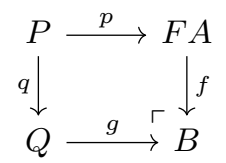

Further, let $(X, Y, u)=\lim _{i \in \mathcal{I}}\left(X_{i}, Y_{i}, u_{i}\right)$ be a $\lambda$-directed colimit in $F \downarrow l_{\mathcal{C}}$, and suppose that $(x: A \rightarrow X, y: B \rightarrow Y)$ is a morphism in $F \downarrow l_{\mathcal{C}}$ :

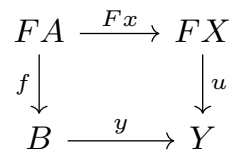

We must show that $(x, y)$ factors through some $\left(X_{i}, Y_{i}, u_{i}\right)$, essentially uniquely.

We first note that, from the colimit diagram $\left(X_{i}, Y_{i}, u_{i}\right)$, we have

$$
\begin{aligned}
& \forall i \leq_{\mathcal{I}} i^{\prime}, \quad X_{i \rightarrow i^{\prime}}: X_{i} \rightarrow X_{i^{\prime}} \quad \text { and } \quad Y_{i \rightarrow i^{\prime}}: Y_{i} \rightarrow Y_{i^{\prime}} \\
& \forall i \in \mathcal{I}, \quad x_{i}: X_{i} \rightarrow X \quad \text { and } \quad y_{i}: Y_{i} \rightarrow Y
\end{aligned}
$$


satisfying $x_{i}=x_{i^{\prime}} \circ X_{i \rightarrow i^{\prime}}, y_{i}=x_{i^{\prime}} \circ Y_{i \rightarrow i^{\prime}}$, and $u \circ F x_{i}=y_{i} \circ u_{i}$. The rest of the proof can be seen by chasing the following diagram:

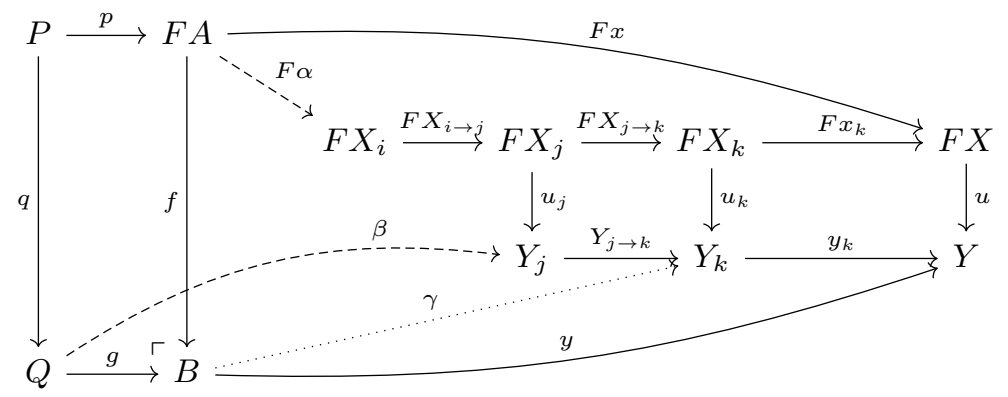

1. By $\lambda$-presentability of $A, x$ factors through some $x_{i}$ via some morphism $\alpha$.

2. By $\lambda$-presentability of $Q, y \circ g$ factors through some $y_{j}$ via some morphism $\beta$.

By directedness of $\mathcal{I}$ we may assume, without loss of generality, that $i \leq_{\mathcal{I}} j$.

3. We thus have two factorizations

$$
\begin{aligned}
y_{j} \circ\left(u_{j} \circ F X_{i \rightarrow j} \circ F \alpha \circ p\right) & =u \circ F x_{j} \circ F X_{i \rightarrow j} \circ F \alpha \circ p \\
& =u \circ F x \circ p \\
& =y \circ f \circ p \\
& =y \circ g \circ q \\
& =y_{j} \circ(\beta \circ q)
\end{aligned}
$$

of a morphism from $P$ to $Y$. By $\lambda$-presentability of $P$, there exists a $k$ (without loss of generality, we may take $j \leq_{\mathcal{I}} k$ ) such that

$$
Y_{j \rightarrow k} \circ u_{j} \circ F X_{i \rightarrow j} \circ F \alpha \circ p=Y_{j \rightarrow k} \circ \beta \circ q
$$

4. Now, by the pushout property of $B$, there exists a $\gamma: B \rightarrow Y_{k}$ that makes $\left(X_{i \rightarrow k} \circ \alpha, \gamma\right)$ into a $F \downarrow$ ld $_{\mathcal{C}}$-morphism from $(A, B, f)$ to $\left(X_{k}, Y_{k}, u_{k}\right)$.

Using $\lambda$-presentability of $A$ and $Q$, together with the fact that $B$ is a pushout, it is easy to verify that the factorization thus obtained is essentially unique.

\section{$3.3 \lambda$-Accessibility of $F \downarrow l_{\mathcal{C}}$}

In this section we show that $\mathcal{P}$ generates all of $F \downarrow l_{\mathcal{C}}$ under $\lambda$-directed colimits.

We first observe that any $(A, B, f) \in F \downarrow \mathbf{l d}_{\mathcal{C}}$ can be written as a $\lambda$-directed colimit

$$
(A, B, f)=\lim _{i \in \mathcal{I}}\left(A_{i}, B_{i}, f_{i}\right)
$$

where $A_{i} \in \mathcal{A}_{0}$. Indeed, since $\mathcal{A}$ is locally $\lambda$-presentable, and thus $\lambda$-accessible, we can write $A=\lim _{i \in \mathcal{I}} A_{i}$ with $a_{i}: A_{i} \rightarrow A$ and each $A_{i} \in \mathcal{A}_{0}$. Now take $\left(A_{i}, B_{i}, f_{i}\right)=\left(A_{i}, B, f \circ F a_{i}\right)$, and observe that $\left((A, B, f),\left\{\left(a_{i}, i d_{B}\right)\right\}_{i \in \mathcal{I}}\right)$ is a colimiting cocone for the diagram $\left\{\left(A_{i}, B_{i}, f_{i}\right)\right\}_{i \in \mathcal{I}}$ with connecting morphisms $\left(a_{i \rightarrow i^{\prime}}, i d_{B}\right):\left(A_{i}, B_{i}, f_{i}\right) \rightarrow\left(A_{i^{\prime}}, B_{i^{\prime}}, f_{i^{\prime}}\right)$. 
Now, the collection of objects of a given category that can be written as $\lambda$ directed colimits of $\lambda$-presentable objects is clearly closed under $\lambda$-directed colimits. It therefore suffices to show that every $(A, B, f) \in F \downarrow \mathrm{ld}_{\mathcal{C}}$ with $A \in \mathcal{A}_{0}$ can be written as a $\lambda$-directed colimit of $\lambda$-presentable objects.

For the rest of this section, let such an $(A, B, f)$ be fixed. Then, using local $\lambda$-presentability of $\mathcal{C}$, we can:

- write $F A=\underline{\lim }_{j \in \mathcal{J}} P_{j}$, where $\mathcal{J}=(J, \leq \mathcal{J})$ is $\lambda$-directed, $P_{j \rightarrow j^{\prime}}: P_{j} \rightarrow P_{j^{\prime}}$ for $j \leq_{\mathcal{J}} j^{\prime}, P_{j} \in \mathcal{C}_{0}$, and $p_{j}: P_{j} \rightarrow F A$ for $j \in J$.

- write $B=\underline{\lim }_{k \in \mathcal{K}} B_{k}$, where $\mathcal{K}=(K, \leq \mathcal{K})$ is $\lambda$-directed, $B_{k \rightarrow k^{\prime}}: B_{k} \rightarrow B_{k^{\prime}}$ for $k \leq_{\mathcal{K}} k^{\prime}, B_{k} \in \mathcal{C}_{0}$, and $b_{k}: B_{k} \rightarrow B$ for $k \in K$.

- use the facts that each $P_{j}$ is $\lambda$-presentable, $B=\lim _{k \in \mathcal{K}} B_{k}$, and $f \circ p_{j}: P_{j} \rightarrow B$ to choose, for each $j \in J, k(j) \in K$ and $q(j): P_{j} \rightarrow B_{k(j)}$ such that the following diagram commutes:

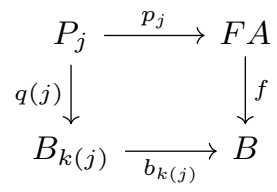

- put, for each $k \geq_{\mathcal{K}} k(j), q(j, k)=B_{k(j) \rightarrow k} \circ q(j): P_{j} \rightarrow B_{k}$, and note that if $k(j) \leq_{\mathcal{K}} k \leq_{\mathcal{K}} k^{\prime}$, then $q\left(j, k^{\prime}\right)=B_{k \rightarrow k^{\prime}} \circ q(j, k)$

With these notations and definitions, we can define the poset $\mathcal{D}=\left(D, \leq_{\mathcal{D}}\right)$ by

$$
\begin{aligned}
D & =\left\{(j, k) \in J \times K \mid k \geq_{\mathcal{K}} k(j)\right\} \\
(j, k) \leq_{\mathcal{D}}\left(j^{\prime}, k^{\prime}\right) & \Longleftrightarrow j \leq_{\mathcal{J}} j^{\prime} \quad \& \quad k \leq_{\mathcal{K}} k^{\prime} \quad \& \quad q\left(j, k^{\prime}\right)=q\left(j^{\prime}, k^{\prime}\right) \circ P_{j \rightarrow j^{\prime}}
\end{aligned}
$$

Reflexivity, antisymmetry, and transitivity of $\leq_{\mathcal{D}}$ are immediate.

Clatm. $\mathcal{D}$ is $\lambda$-directed.

Proof In the remainder of this paper, if $\mathcal{X}=(X, \leq \mathcal{X})$ is a poset, $x \in X$, and $S \subseteq X$ we write $x \geq \mathcal{X} S$ to indicate that $x$ is an upper bound for $S$.

Suppose that $\left\{\left(j_{i}, k_{i}\right) \mid i \in I\right\} \subseteq D$, with $|I|<\lambda$. Using $\lambda$-directedness of $\mathcal{J}$ and $\mathcal{K}$, let $j^{*} \geq_{\mathcal{J}}\left\{j_{i} \mid i \in I\right\}, k_{0}^{*} \geq_{\mathcal{K}}\left\{k_{i} \mid i \in I\right\}$, and $k_{1}^{*} \geq_{\mathcal{K}}\left\{k_{0}^{*}, k\left(j^{*}\right)\right\}$. Then for each $i \in I$, we have two factorizations of $f \circ p_{j_{i}}$ through $b_{k_{1}^{*}}$ :

$$
b_{k_{1}^{*}} \circ q\left(j_{i}, k_{1}^{*}\right)=f \circ p_{j_{i}}=b_{k_{1}^{*}} \circ q\left(j^{*}, k_{1}^{*}\right) \circ P_{j_{i} \rightarrow j^{*}}
$$

Since $P_{j_{i}}$ is $\lambda$-presentable, the two morphisms must be coequalized by some connecting map. So let $k_{2}^{*}(i) \geq \mathcal{K} k_{1}^{*}$ be such that

$$
B_{k_{1}^{*} \rightarrow k_{2}^{*}(i)} \circ q\left(j_{i}, k_{1}^{*}\right)=B_{k_{1}^{*} \rightarrow k_{2}^{*}(i)} \circ q\left(j^{*}, k_{1}^{*}\right) \circ P_{j_{i} \rightarrow j^{*}}
$$

That is,

$$
q\left(j_{i}, k_{2}^{*}(i)\right)=q\left(j^{*}, k_{2}^{*}(i)\right) \circ P_{j_{i} \rightarrow j^{*}}
$$

Finally, let $k^{*} \geq_{\mathcal{K}}\left\{k_{2}^{*}(i) \mid i \in I\right\}$. Then, for each $i$, post-composing both sides of the preceding equation with $B_{k_{2}^{*}(i) \rightarrow k^{*}}$ yields

$$
\begin{aligned}
q\left(j_{i}, k^{*}\right) & =B_{k_{2}^{*}(i) \rightarrow k^{*}} \circ q\left(j_{i}, k_{2}^{*}(i)\right) \\
& =B_{k_{2}^{*}(i) \rightarrow k^{*}} \circ q\left(j^{*}, k_{2}^{*}(i)\right) \circ P_{j_{i} \rightarrow j^{*}}=q\left(j^{*}, k^{*}\right) \circ P_{j_{i} \rightarrow j^{*}}
\end{aligned}
$$

We thus have that $\left(j^{*}, k^{*}\right) \geq_{\mathcal{D}}\left\{\left(j_{i}, k_{i}\right) \mid i \in I\right\}$. 
Now note that $D$ is cofinal in the poset $\mathcal{J} \times \mathcal{K}$. Indeed, if $(j, k) \in J \times K$, choose $k^{\prime} \geq\{k, k(j)\}$ by $\lambda$-directedness of $\mathcal{K}$. Then $\left(j, k^{\prime}\right) \in D$ and $\left(j, k^{\prime}\right) \geq \mathcal{J} \times \mathcal{K}(j, k)$.

To define the diagram representing $(A, B, f)$ as a $\mathcal{D}$-indexed colimit of $\lambda$ presentable objects, we first define a $\mathcal{D}$-indexed diagram of spans in $\mathcal{C}$. Let $\mathcal{S}=$ $\{a \stackrel{l}{\longleftarrow} b \stackrel{r}{\longrightarrow} c\}$ be the "walking span", i.e., the index category for pushout diagrams. Consider the diagram $G: \mathcal{D} \rightarrow \mathcal{C}^{\mathcal{S}}$, where

$$
\begin{aligned}
& G(j, k) \quad=\quad B_{k} \stackrel{q(j, k)}{\longleftarrow} P_{j} \stackrel{p_{j}}{\longrightarrow} F A \\
& G\left(\begin{array}{c}
(j, k) \\
\downarrow \leq \mathcal{D} \\
\left(j^{\prime}, k^{\prime}\right)
\end{array}\right)=\quad \begin{array}{ccc}
B_{k} \stackrel{q(j, k)}{\stackrel{p^{\prime}}{\leftrightarrows}} & P_{j} \stackrel{p_{j}}{\longrightarrow} F A \\
B_{k^{\prime}} \stackrel{q\left(j^{\prime}, k^{\prime}\right)}{\longleftarrow} & P_{j^{\prime}} \stackrel{p_{j^{\prime}}}{\longrightarrow} F A
\end{array}
\end{aligned}
$$

By direct computation of colimits in functor categories and cofinality of $D$ in $\mathcal{J} \times \mathcal{K}, \lim _{(j, k) \in \mathcal{D}} G(j, k)$ is

$$
G^{*}=\{B \longleftarrow f(F A=F A\}
$$

with cocone morphisms $\left\{\left(b_{k}, p_{j}, i d_{F A}\right): G(j, k) \Rightarrow G^{*} \mid(j, k) \in D\right\}$.

For each $(j, k) \in D, \lim _{s \in \mathcal{S}} G(j, k)(s)$ is the pushout

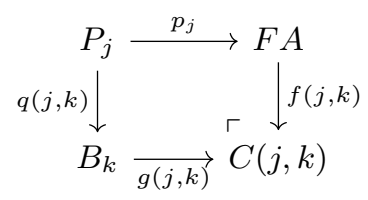

whose right side defines a $\lambda$-presentable object $(A, C(j, k), f(j, k): F A \rightarrow C(j, k))$ of $F \downarrow l d_{\mathcal{C}}$ whose membership in $\mathcal{P}$ is witnessed by $\left(A, P_{j}, B_{k}, p_{j}, q(j, k)\right) \in \mathcal{W}$. We therefore define the diagram $H: \mathcal{D} \rightarrow$ $\mathcal{P}$ by projection on right sides, i.e., $H(j, k)=(A, C(j, k), f(j, k))$, as in (1). The action of $H$ on morphisms is depicted in the cube displayed on the right. For $(j, k) \leq_{\mathcal{D}}\left(j^{\prime}, k^{\prime}\right)$, the pair $\left(i d_{A}, h\right)$, where $h$ is obtained from the fact that $C(j, k)$ is a pushout, gives a morphism in $F \downarrow \operatorname{ld}_{\mathcal{C}}$ from $(A, C(j, k), f(j, k))$ to $\left(A, C\left(j^{\prime}, k^{\prime}\right), f\left(j^{\prime}, k^{\prime}\right)\right)$.

Since colimits commute with one another, the colimit of these pushouts is the pushout of the colimits, i.e.,

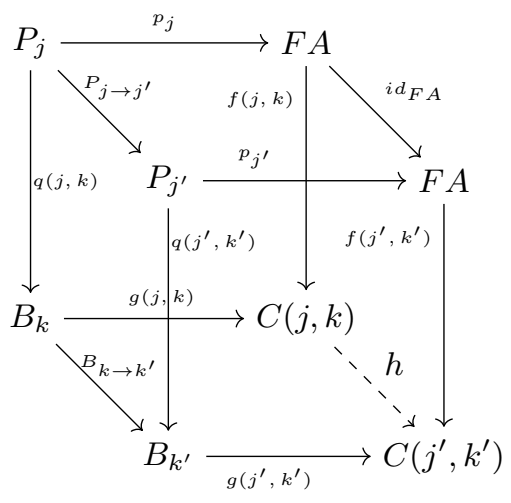

$$
\lim _{(j, k) \in \mathcal{D}} \lim _{s \in \mathcal{S}} G(j, k)(s)=\lim _{s \in \mathcal{S}} \lim _{(j, k) \in \mathcal{D}} G(j, k)(s)=\lim _{s \in \mathcal{S}} G^{*}(s)
$$


Since the pushout of $f$ along the identity is $f$, the last expression in this sequence of equations is just the pushout

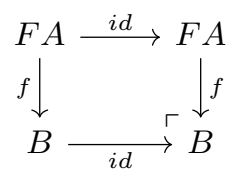

Taking the projection on the right side then gives that

$$
\lim _{(j, k) \in \mathcal{D}} H(j, k)=(F A \stackrel{f}{\longrightarrow} B)
$$

i.e.,

$$
\lim _{(j, k) \in \mathcal{D}}(A, C(j, k), f(j, k))=(A, B, f)
$$

This shows that $(A, B, f)$ is indeed a $\mathcal{D}$-indexed colimit of $\lambda$-presentable objects in $F \downarrow l_{\mathcal{C}}$, and thus completes the proof of $\lambda$-accessibility of $F \downarrow l d_{\mathcal{C}}$.

This completes the proof of Proposition 4.

\section{An Open Question}

In this paper we observed that, for a $\lambda$-accessible functor $F: \mathcal{A} \rightarrow \mathcal{C}$ between locally $\lambda$-presentable categories, both categories $\operatorname{Id}_{\mathcal{C}} \downarrow F$ and $F \downarrow I_{\mathcal{C}}$ are locally $\lambda$ presentable. One naturally wonders how far these results can be extended.

For the general case of the comma category between two $\lambda$-accessible functors between locally $\lambda$-presentable categories, local $\lambda$-presentability fails whenever cocompleteness fails. By Proposition 5, cocompleteness would be assured if the domain functor were cocontinuous. The following question therefore suggests a natural line of inquiry which appears to be beyond the techniques of this paper:

Question. If $\mathcal{A}, \mathcal{B}$, and $\mathcal{C}$ are locally $\lambda$-presentable categories, $F_{1}: \mathcal{A} \rightarrow \mathcal{C}$ and $F_{2}: \mathcal{B} \rightarrow \mathcal{C}$ are $\lambda$-accessible functors, and $F_{1}$ is cocontinuous, then is $F_{1} \downarrow F_{2}$ locally $\lambda$-presentable?

\section{References}

1. Adámek, J. and Rosický, J., Locally Presentable and Accessible Categories. Cambridge University Press, 1994

2. Kelly, G. M., A Unified Treatment of Transfinite Constructions for Free Algebras, Free Monoids, Colimits, Associated Sheaves, and so on. Bulletin of the Australian Mathematical Society 22 (1980), pp. 1-83. 